\title{
Diabetes mellitus deteriorates left ventricular deformation in acromegaly - analysis from the three-dimensional speckle-tracking echocardiographic MAGYAR-Path study
}

\author{
Attila Nemes ${ }^{1}$, Árpád Kormányos ${ }^{1}$, Péter Domsik ${ }^{1}$, Anita Kalapos ${ }^{1}$, Nándor Gyenes ${ }^{1}$, Csaba Lengyel ${ }^{2}$, \\ Zsuzsanna Valkusz ${ }^{2}$ \\ ${ }^{1}$ 2nd Department of Medicine and Cardiology Center, Medical Faculty, Albert Szent-Györgyi Clinical Center, University of Szeged, Szeged, \\ Hungary; ${ }^{2}$ sst Department of Medicine, Medical Faculty, Albert Szent-Györgyi Clinical Center, University of Szeged, Szeged, Hungary
}

Correspondence to: Attila Nemes, MD, PhD, DSc, FESC. 2nd Department of Medicine and Cardiology Center, Medical Faculty, Albert Szent-Györgyi Clinical Center, University of Szeged, H-6725 Szeged, Semmelweis street 8, P.O. Box 427, Hungary. Email: nemes.attila@med.u-szeged.hu.

\begin{abstract}
Acromegaly and diabetes mellitus (DM) separately are associated with cardiovascular alterations and modified left ventricular (LV) deformation, which can be quantitatively assessed by three-dimensional speckle-tracking echocardiography (3DSTE). The present study aimed to assess the relationship between $\mathrm{LV}$ deformation and twist in the presence of DM in acromegaly. Thirty-seven acromegaly patients were involved in the study, but due to insufficient image quality 13 have to be excluded from analysis. Therefore, the present study comprised 24 acromegaly patients, from which 5 had DM. Their data was compared to 35 age- and gender matched healthy controls. Complete two-dimensional Doppler echocardiography extended with 3DSTE were performed in all patients and controls. Only non-diabetic acromegaly cases had increased global and mean segmental LV radial strain, diabetic acromegaly patients had similar LV radial strain as compared to that of healthy subjects. Other LV strain parameters did not show any difference between diabetic and non-diabetic acromegaly patients. While $\mathrm{LV}$ basal rotation was similar among the groups, LV apical rotation was reduced in both diabetic and non-diabetic patients as compared to healthy subjects. However, diabetic acromegaly patients had tendentiously higher values. The ratio of absence of LV twist called as LV rigid body rotation was similar between the groups examined. Diabetes mellitus deteriorates left ventricular deformation in acromegaly.
\end{abstract}

Keywords: Acromegaly; diabetes mellitus; left ventricular; deformation

Submitted Jan 27, 2020. Accepted for publication Jul 01, 2020.

doi: 10.21037/qims-20-159

View this article at: http://dx.doi.org/10.21037/qims-20-159

\section{Introduction}

Acromegaly is a chronic hormonal disease characterized by chronic overproduction of growth hormone $(\mathrm{GH})$ caused by a benign pituitary tumor (1). Wide range of cardiovascular alterations could be detected in acromegaly including hypertension, left ventricular (LV) hypertrophy and dysfunction, valvular abnormalities and arrhythmias $(2,3)$. Diabetes mellitus $(\mathrm{DM})$ is a strong cardiovascular risk factor known to be able to modify $L V$ function (4). Three-dimensional (3D) speckle-tracking echocardiography (3DSTE) is a new non-invasive imaging technique, which is able to quantify $\mathrm{LV}$ deformation including $\mathrm{LV}$ strains, which are objective features of LV contractility (5). Moreover, $\mathrm{LV}$ is able to wring itself called $\mathrm{LV}$ twist, which can be characterized by different $\mathrm{LV}$ rotational parameters (6). The present study aimed to assess the relationship between $\mathrm{LV}$ deformation and twist in the presence of DM in acromegaly. 


\section{Methods}

\section{Patient population}

Thirty-seven acromegaly patients were involved in the study, but due to insufficient image quality 13 have to be excluded from analysis. Therefore, the present study comprised 24 acromegaly patients, from which 5 had DM. Bromocriptine and long-acting somatostatine analogues were used in 4 and 4 patients, respectively, while hypophysectomy was performed in 6 patients previously in the presence of active acromegaly. In the inactive acromegaly group, 2 and 3 patients were taking bromocriptine and/or long-acting somatostatine analogues, respectively, while 4 patients had previous successful hypophysectomy. Their data was compared to 35 age- and gender matched healthy controls. Diagnosis of DM and acromegaly was based on current guidelines $(4,7)$. The present study is part of the MAGYARPath Study (Motion Analysis of the heart and Great vessels bY three-dimensionAl speckle-tRacking echocardiography in Pathological cases), which was organised to assess diagnostic and prognostic value of different 3DSTE-derived parameters ('magyar' means 'Hungarian' in Hungarian language). The study conformed to the provisions of the Declaration of Helsinki (as revised in 2013). The study was approved by institutional ethics committee of the University of Szeged (No.: 71/2011) and informed consent was taken from all the patients.

\section{Two-dimensional Doppler echocardiography}

Complete two-dimensional (2D) Doppler transthoracic echocardiography was performed in all patients and healthy volunteers by a Toshiba Artida ${ }^{\mathrm{TM}}$ echocardiographic tool (Toshiba Medical Systems, Tokyo, Japan) using a PST30 SBP $(1-5 \mathrm{MHz})$ phased-array transducer according the guidelines by the same observer (Á.K.) (8).

\section{Three-dimensional speckle-tracking echocardiography}

The same Toshiba Artida ${ }^{\mathrm{TM}}$ echocardiography machine with a PST-25SX matrix-array transducer (Toshiba Medical Systems, Tokyo, Japan) was used for 3DSTE following our routine practice $(9,10)$. Shortly, a full volume 3D dataset was acquired during a single breath-hold from 6 wedgeshaped subvolumes during 6 cardiac cycles. These datasets were analysed offline using a 3D Wall Motion Tracking software version 2.7 (Toshiba Medical Systems, Tokyo, Japan). During analysis, a 3D LV cast was created using apical four-chamber $(\mathrm{AP} 4 \mathrm{CH})$ and two-chamber $(\mathrm{AP} 2 \mathrm{CH})$ views and 3 cross-sectional views. Using this $3 \mathrm{D}$ LV model, several LV volumetric, strain and rotational parameters were calculated. For each segment, radial (thickening and thinning of the segment, RS), longitudinal (lengthening and shortening of the segment, LS), circumferential (widening and narrowing of the segment, CS), area (combination of LS and CS, AS) and 3D strain (combination of RS, LS and CS, 3DS) were calculated. Moreover, LV basal and apical rotation and $\mathrm{LV}$ twist were also measured.

\section{Statistical analysis}

All continuous variables were presented as mean \pm standard deviation, while categorical data were demonstrated as frequencies and percentages (\%). P values $<0.05$ were considered to be statistically significant. For comparison of numerical and categorical variables, 2 -tailed Student's $t$ test and Fisher's exact test was performed, respectively. For datasets not normally distributed, we used MannWhitney-Wilcoxon test to assess statistical significance. For the statistical analysis, RStudio was used (RStudio Team, RStudio: Integrated Development for R. RStudio, Inc., Boston, MA, 2015). For offline data extraction and analysis, a commercial software package was used (MATLAB 8.6, The MathWorks Inc., Natick, MA, 2015).

\section{Results}

Classic cardiovascular risk factors, activity of acromegaly and 2D echocardiographic data in all, diabetic and non-diabetic acromegaly patients and healthy controls are presented in Table 1. Diabetic patients were older and had higher ratio of hypertension as compared to non-diabetic subjects. Higher LA and LV end-diastolic dimensions, thickened LV posterior wall and interventricular septum and diastolic dysfunction could be demonstrated in acromegaly subjects. No differences could be detected in the routine $2 \mathrm{D}$ echocardiographic data between diabetic and non-diabetic acromegaly cases.

Only non-diabetic acromegaly cases had increased global and mean segmental LV-RS, diabetic acromegaly patients had similar LV-RS as compared to that of healthy subjects (Table 2). Other LV strain parameters did not show any difference between diabetic and non-diabetic acromegaly patients. While LV basal rotation was similar among the groups, LV apical rotation was reduced in both diabetic and non-diabetic acromegaly patients as compared to healthy subjects. However, diabetic acromegaly patients 
Table 1 Risk factors and two-dimensional echocardiographic data of controls and acromegaly patients

\begin{tabular}{|c|c|c|c|c|}
\hline Data & Controls $(n=35)$ & $\begin{array}{l}\text { All acromegaly patients } \\
\qquad(n=24)\end{array}$ & $\begin{array}{l}\text { Acromegaly patients with } \\
\qquad \text { DM }(n=5)\end{array}$ & $\begin{array}{l}\text { Acromegaly patients without } \\
\qquad D M(n=19)\end{array}$ \\
\hline \multicolumn{5}{|l|}{ Risk factors } \\
\hline Male gender & $15(43 \%)$ & 7 (29\%) & $1(20 \%)$ & $6(32 \%)$ \\
\hline Hypertension & 0 & $14(58 \%)^{*}$ & $5(100 \%)^{*}$ & $9(47 \%)^{\star \dagger}$ \\
\hline Hyperlipidaemia & 0 & $11(46 \%)^{*}$ & $3(60 \%)^{\star}$ & $8(42 \%)^{\star}$ \\
\hline Active acromegaly & 0 & $13(54 \%)^{*}$ & $3(60 \%)^{*}$ & $10(53 \%)^{*}$ \\
\hline \multicolumn{5}{|l|}{ Two-dimensional echocardiography } \\
\hline LA diameter (mm) & $38.7 \pm 4.3$ & $42.2 \pm 6.3^{*}$ & $46.1 \pm 7.5$ & $41.2 \pm 5.7$ \\
\hline LV end-systolic diameter (mm) & $31.6 \pm 3.0$ & $31.7 \pm 4.8$ & $31.9 \pm 4.0$ & $31.6 \pm 5.1$ \\
\hline LV end-systolic volume (mL) & $37.9 \pm 8.9$ & $42.0 \pm 15.0$ & $42.0 \pm 11.2$ & $42.0 \pm 16.2$ \\
\hline Interventricular septum (mm) & $9.4 \pm 1.4$ & $10.4 \pm 1.6^{*}$ & $10.5 \pm 0.7^{*}$ & $10.4 \pm 1.7^{*}$ \\
\hline LV posterior wall (mm) & $9.7 \pm 1.5$ & $10.9 \pm 1.7^{*}$ & $11.5 \pm 1.2^{*}$ & $10.8 \pm 1.8^{*}$ \\
\hline LV ejection fraction (\%) & $66.0 \pm 5.0$ & $67.7 \pm 7.3$ & $67.9 \pm 6.2$ & $67.6 \pm 7.8$ \\
\hline $\mathrm{E}(\mathrm{cm} / \mathrm{s})$ & $71.4 \pm 18.6$ & $65.5 \pm 14.3$ & $74.0 \pm 22.0$ & $63.7 \pm 12.2$ \\
\hline $\mathrm{A}(\mathrm{cm} / \mathrm{s})$ & $64.5 \pm 3.5$ & $79.0 \pm 16.4$ & $84.4 \pm 14.4$ & $77.8 \pm 16.9$ \\
\hline $\mathrm{E} / \mathrm{A}$ & $1.07 \pm 0.31$ & $0.85 \pm 0.21^{*}$ & $0.88 \pm 0.27$ & $0.85 \pm 0.21^{*}$ \\
\hline
\end{tabular}

${ }^{*} \mathrm{P}<0.05$ vs. Controls; ${ }^{\dagger} \mathrm{P}<0.05$ vs. acromegaly patients with $\mathrm{DM}$. LA, left atrium; LV, left ventricle; E, early transmitral flow velocity; A, late transmitral flow velocity; DM, diabetes mellitus.

had tendentiously higher values. The ratio of absence of $\mathrm{LV}$ twist called as $\mathrm{LV}$ rigid body rotation (RBR) was similar between the groups examined.

\section{Discussion}

Both acromegaly and DM are known to be associated with severe, but different $\mathrm{LV}$ deformation abnormalities including strain and rotational changes (4,9-11). Aortic stiffness is increased in both diseases $(12,13)$, and the presence of DM facilitates aortic stiffening in acromegaly (14). Therefore, a question has been arisen, whether it has any effect on otherwise supranormal LV deformation (strain) in acromegaly (10).

In a recent study, severe abnormalities in $L V$ twisting mechanism could be detected in acromegaly including significant reduction in LV apical rotation and twist (9).
Moreover, absence of LV twist called as LV-RBR was found in $20 \%$ of acromegaly patients $(9,15)$. These significant abnormalities could be theoretically explained by acromegaly-related hormonal changes and their effect on fiber structure. Although these abnormalities are present, LV systolic (pumping) function is normal in these patients due to compensatory increased LV contractility represented by increased LV-RS, which was found to be related to disease activity (10). These studies confirmed significant deterioration of $\mathrm{LV}$ twisting mechanism with a compensatory increased LV contractility, giving us deeper insight into the acromegalic cardiomyopathy. With the finding of the present study, this knowledge was widened demonstrating a relative reduction in LV-RS in acromegaly patients with DM from hypercontractility to the level of LV-RS seen in healthy, non-acromegalic, non-diabetic subjects as compared to non-diabetic cases. Moreover, LV 
Table 2 Three-dimensional speckle-tracking echocardiographic data of controls and acromegaly patients

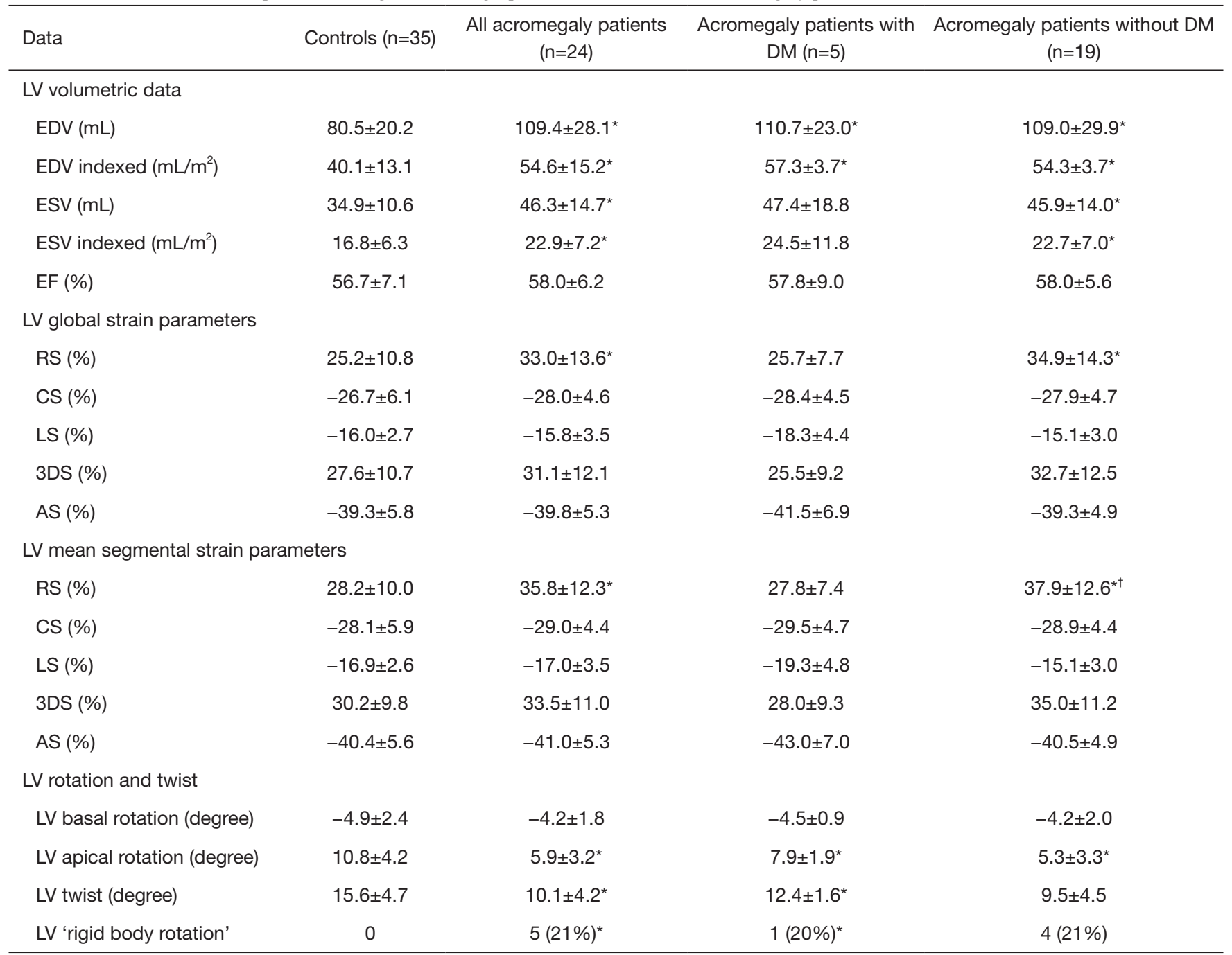

${ }^{*} \mathrm{P}<0.05$ vs. Controls; ${ }^{\dagger} \mathrm{P}<0.05$ vs. acromegaly patients with $\mathrm{DM}$. EDD, end-diastolic diameter; EDV, end-systolic volume; EF, ejection fraction; RS, radial strain; LS, longitudinal strain; CS, circumferential strain; 3DS, three-dimensional strain; AS, area strain; LV, left ventricle; DM, diabetes mellitus.

apical rotation was found to be lower in acromegaly patients with tendentiously higher values in diabetic acromegaly patients as compared to that of non-diabetic acromegaly cases suggesting a possible compensatory mechanism. Interestingly, the ratio of LV-RBR did not differ between diabetic and non-diabetic acromegaly patients suggesting no effects of DM on the development of LV-RBR. All diabetic patients had hypertension as well, which could affect results. However, further studies with more acromegaly patients are warranted to confirm our findings with other methods. Finally, it can be concluded that DM deteriorates LV deformation in acromegaly.

\section{Acknowledgments}

Funding: None.

\section{Footnote}

Conflicts of Interest: All authors have completed the ICMJE uniform disclosure form (available at http://dx.doi. org/10.21037/qims-20-159). AN serves as an unpaid editorial board member of Quantitative Imaging in Medicine and Surgery. The other authors have no conflicts of interest to declare. 
Ethical Statement: The authors assert that all procedures contributing to this work comply with the ethical standards of the relevant national guidelines on human experimentation and with the Helsinki Declaration of 1975 , as revised in 2013, and has been approved by the institutional committee of the University of Szeged. The study was approved by institutional ethics committee of the University of Szeged (No.: 71/2011) and informed consent was taken from all the patients. Written informed consent was obtained from the patient for publication of this study and any accompanying images. A copy of the written consent is available for review by the Editor-in-Chief of this journal.

Open Access Statement: This is an Open Access article distributed in accordance with the Creative Commons Attribution-NonCommercial-NoDerivs 4.0 International License (CC BY-NC-ND 4.0), which permits the noncommercial replication and distribution of the article with the strict proviso that no changes or edits are made and the original work is properly cited (including links to both the formal publication through the relevant DOI and the license). See: https://creativecommons.org/licenses/by-nc-nd/4.0/.

\section{References}

1. Sanno N, Teramoto A, Osamura RY, Horvath E, Kovacs K, Lloyd RV, Scheithauer BW. Pathology of pituitary tumors. Neurosurg Clin N Am 2003;14:25-39.

2. Clayton RN. Cardiovascular function in acromegaly. Endocr Rev 2003;24:272-7.

3. Ramos-Leví AM, Marazuela M. Cardiovascular comorbidities in acromegaly: an update on their diagnosis and management. Endocrine 2017;55:346-59.

4. Wierzbowska-Drabik K, Trzos E, Kurpesa M, Rechcinski T, Miskowiec D, Cieslik-Guerra U, Uznanska-Loch B, Sobczak M, Kasprzak JD. Diabetes as an independent predictor of left ventricular longitudinal strain reduction at rest and during dobutamine stress test in patients with significant coronary artery disease. Eur Heart J Cardiovasc Imaging 2018;19:1276-86.

5. Nemes A, Kalapos A, Domsik P, Forster T. Threedimensional speckle-tracking echocardiography -- a further step in non-invasive three-dimensional cardiac imaging. Orv Hetil 2012;153:1570-7.

6. Nemes A, Kalapos A, Domsik P, Forster T. Left ventricular rotation and twist of the heart. Clarification of some concepts. Orv Hetil 2012;153:1547-51.
7. Melmed S. Medical progress: Acromegaly. N Engl J Med 2006;355:2558-73.

8. Lang RM, Badano LP, Mor-Avi V, Afilalo J, Armstrong A, Ernande L, Flachskampf FA, Foster E, Goldstein SA, Kuznetsova T, Lancellotti P, Muraru D, Picard MH, Rietzschel ER, Rudski L, Spencer KT, Tsang W, Voigt JU. Recommendations for cardiac chamber quantification by echocardiography in adults: an update from the American Society of Echocardiography and the European Association of Cardiovascular Imaging. Eur Heart J Cardiovasc Imaging 2015;16:233-70.

9. Kormányos Á, Domsik P, Kalapos A, Orosz A, Lengyel C, Valkusz Z, Trencsányi A, Forster T, Nemes A. Left ventricular twist is impaired in acromegaly: Insights from the three-dimensional speckle tracking echocardiographic MAGYAR-Path Study. J Clin Ultrasound 2018;46:122-8.

10. Kormányos Á, Domsik P, Kalapos A, Gyenes N, Valkusz Z, Lengyel C, Forster T, Nemes A. Active acromegaly is associated with enhanced left ventricular contractility: Results from the three-dimensional speckle-tracking echocardiographic MAGYAR-Path Study. Rev Port Cardiol 2020;39:189-96.

11. Pokharel P, Yoon AJ, Bella JN. Non invasive measurement and clinical relevance of myocardial twist and torsion. Expert Rev Cardiovasc Ther 2014;12:1305-15.

12. Nemes A, Gavallér H, Csajbók E, Julesz J, Forster T, Csanády $M$. Aortic stiffness is increased in acromegaly- a transthoracic echocardiographic study. Int J Cardiol 2008;124:121-3.

13. Nemes A, Forster T, Lengyel C, Csanády M. Reduced aortic distensibility and coronary flow velocity reserve in diabetes mellitus patients with a negative coronary angiogram. Can J Cardiol 2007;23:445-50.

14. Nemes A, Gavallér H, Csajbók E, Lengyel C, Forster T, Csanády M. Does diabetes mellitus facilitate aortic stiffening in acromegaly? Diabetes Res Clin Pract 2007;78:e7-8.

15. Nemes A, Kormányos Á, Domsik P, Kalapos A, Lengyel C, Valkusz Z, Forster T. Left ventricular 'rigid body rotation' in a patient with acromegaly (from the MAGYAR-Path Study). Quant Imaging Med Surg 2017;7:378-9.

Cite this article as: Nemes A, Kormányos Á, Domsik P, Kalapos A, Gyenes N, Lengyel C, Valkusz Z. Diabetes mellitus deteriorates left ventricular deformation in acromegalyanalysis from the three-dimensional speckle-tracking echocardiographic MAGYAR-Path study. Quant Imaging Med Surg 2021;11(1):410-414. doi: 10.21037/qims-20-159 